\title{
On bipartite powers of bigraphs
}

\author{
Yoshio Okamoto $\|^{1} \quad$ Yota Otachi" $\|^{2} \quad$ Ryuhei Uehara ${ }^{2}$
}

${ }^{1}$ Department of Communication Engineering and Informatics, Graduate School of Informatics and Engineering, University of Electro-Communications, Chofugaoka 1-5-1, Chofu, Tokyo, 182-8585 Japan.

${ }^{2}$ School of Information Science, Japan Advanced Institute of Science and Technology, Asahidai 1-1, Nomi, Ishikawa 923-1292, Japan.

received $11^{\text {th }}$ April 2012, accepted $16^{\text {th }}$ July 2012.

The notion of graph powers is a well-studied topic in graph theory and its applications. In this paper, we investigate a bipartite analogue of graph powers, which we call bipartite powers of bigraphs. We show that the classes of bipartite permutation graphs and interval bigraphs are closed under taking bipartite power. We also show that the problem of recognizing bipartite powers is NP-complete in general.

Keywords: Bipartite power, Interval bigraph, Bipartite permutation graph, Closure property, NP-completeness

\section{Introduction}

For a positive integer $k$, the $k$ th power $G^{k}$ of a graph $G$ has the same vertex set as $G$, and two vertices are adjacent in $G^{k}$ if and only if their distance in $G$ is at most $k$. If $H^{k}=G$, then $H$ is called a $k$ th root of $G$. (In general, $k$ th roots of a graph are not unique and do not necessarily exist.) The concept of graph powers has been extensively studied in graph theory and its algorithmic applications (see Prisner (1995) and the references therein). It is known that several important graph classes are closed under the power operation. That is, for some graph class $\mathcal{C}, G \in \mathcal{C}$ implies $G^{k} \in \mathcal{C}$ as well. The recognition problem has also been studied for graph powers. It was shown that recognizing graph powers is NP-complete in general while it is solvable in polynomial time for some special graph classes (Chang et al. (2006); Farzad et al. (2009); Kearney and Corneil (1998); Lau (2006); Lau and Corneil (2004); Le and Nguyen (2010); Lin and Skiena (1995); Motwani and Sudan (1994)).

A graph is bipartite if its vertices can be partitioned into two parts in such a way that the endpoints of every edge belong to different parts. Bipartite graphs are also known as bigraphs. It is known that the class of bigraphs coincides with the class of graphs with no cycle of odd length. If a bigraph is connected and has at least three vertices, then clearly its $k$ th power (for $k \geq 2$ ) has a cycle of length three. Hence, the class of bigraphs is not closed under the power operation. Furthermore, any non-trivial subclass of the class of bigraphs is not closed under the power operation.

\footnotetext{
†Email: okamotoy@uec.ac.jp.

¥Email: otachi@jaist.ac.jp.

§Email: uehara@jaist.ac.jp.
} 
Chandran et al. (2011) introduced a bipartite analogue of graph powers. For odd $k \in \mathbb{Z}^{+}$, the $k$ th bipartite power $G^{[k]}$ of a bigraph $G$ has the same vertex set as $G$, and two vertices are adjacent in $G^{[k]}$ if and only if their distance in $G$ is at most $k$ and odd. It is easy to see that $G^{[k]}$ is also bipartite. They showed that for any tree $T$ and for any odd $k, T^{[k]}$ is a chordal bipartite graph. They used this fact to prove that chordal bipartite graphs can have arbitrarily large boxicity. Recently, Chandran and Mathew (2012) have strengthened the closure-property result by showing that the class of chordal bipartite graphs is closed under the bipartite power operation.

In this paper, we complement the known closure-property results by proving that some subclasses of the class of chordal bipartite graphs are closed under the bipartite power operation. Note that the closure property of a graph class $\mathcal{C}$ under the bipartite power operation is not implied by the closure property of a superclass of $\mathcal{C}$, and vice versa. We also focus on the computational complexity of the problem for recognizing the $k$ th bipartite power graphs. We prove that given a bipartite graph $G$, the problem of determining whether there exists a bipartite graph $H$ such that $G=H^{[k]}$ is NP-complete for any fixed odd $k \geq 3$.

\section{Preliminaries}

In this paper, graphs are finite, simple, and undirected. We denote by $N_{G}(v)$ the neighborhood of a vertex $v$ in a graph $G$. We denote by $\operatorname{dist}_{G}(u, v)$ the distance between $u$ and $v$ in $G$.

A graph $G=(V, E)$ with $V=\{1,2, \ldots, n\}$ is a permutation graph if there is a permutation $\pi$ over $V$ such that $\{i, j\} \in E(G)$ if and only if $(i-j)(\pi(i)-\pi(j))<0$. A graph is a bipartite permutation graph if it is bipartite and a permutation graph. A bi-interval representation of a bigraph $G=(U, V ; E)$ is a pair $\left(I_{U}, I_{V}\right)$ of sets of closed intervals such that $I_{U}=\left\{\left[\ell_{u}, r_{u}\right] \mid u \in U\right\}$ and $I_{V}=\left\{\left[\ell_{v}, r_{v}\right] \mid v \in V\right\}$, and $\{u, v\} \in E(G)$ if and only if $\left[\ell_{u}, r_{u}\right] \cap\left[\ell_{v}, r_{v}\right] \neq \emptyset$ for $u \in U$ and $v \in V$. A bi-interval representation $\left(I_{U}, I_{V}\right)$ is unit if for each interval $[\ell, r] \in I_{U} \cup I_{V}, r-\ell=1$. A bigraph is an interval bigraph if it has a bi-interval representation. An interval bigraph is a unit interval bigraph if it has a unit bi-interval representation. A bigraph is a chordal bipartite graph if every induced cycle is of length four. We denote the classes of bipartite permutation graphs, unit interval bigraphs, interval bigraphs, and chordal bipartite graphs by $\mathrm{BP}, \mathrm{UIB}, \mathrm{IB}$, and $\mathrm{CB}$, respectively. The following relations among these classes are known.

Theorem 2.1 (Hell and Huang (2004); Müller (1997)) $B P=U I B \subset I B \subset C B$.

\section{Closure properties of bipartite powers of bigraphs}

It is known that several important (non-bipartite) graph classes are closed under the power operation. For example, the classes of unit interval graphs, interval graphs, strongly chordal graphs are closed under the $k$ th power for any $k$ (Chen and Chang (2001); Dahlhaus and Duchet (1987); Lubiw (1987); Raychaudhuri (1987, 1992)). On the other hand, few things are known on bipartite powers of bigraphs. To the best of our knowledge, the following is the only known closure property.

Theorem 3.1 (Chandran and Mathew (2012)) If $G$ is a chordal bipartite graph, then so is $G^{[k]}$ for any odd $k \in \mathbb{Z}^{+}$.

In this section, we show that the closure property holds also for smaller graph classes.

Theorem 3.2 If $G$ is an interval bigraph, then so is $G^{[k]}$ for any odd $k \in \mathbb{Z}^{+}$.

Theorem 3.3 If $G$ is a bipartite permutation graph, then so is $G^{[k]}$ for any odd $k \in \mathbb{Z}^{+}$. 
We note that the closure property is not hereditary for the graph classes. That is, Theorem 3.1 does not imply Theorem 3.2, and Theorem 3.2 does not imply Theorem 3.3 .

\subsection{Bipartite powers of interval bigraphs}

Now we prove Theorem 3.2 Let $G=(U, V ; E)$ be an interval bigraph, and $\left(I_{U}, I_{V}\right)$ be a bi-interval representation of $G$. For odd $k \in \mathbb{Z}^{+}$and $I_{w} \in I_{U} \cup I_{V}$, we form a new interval $I_{w}(k)=\left[\ell_{w}, r_{w}(k)\right]$, where

$$
r_{w}(k)=\max \left\{l_{x} \mid \operatorname{dist}_{G}(w, x) \text { is odd and at most } k\right\} .
$$

This extension of intervals is inspired by the proof of Agnarsson et al. (2000), who showed that the $k$ th power of an interval graph is also an interval graph. Note that $I_{u} \cap I_{v} \neq \emptyset$ if and only if $\max \left\{\ell_{u}, \ell_{v}\right\} \leq$ $\min \left\{r_{u}, r_{v}\right\}$. The following two lemmas show that for $u \in U, v \in V$, and odd $k \in \mathbb{Z}^{+}, I_{u}(k) \cap I_{v}(k) \neq \emptyset$ if and only if $\operatorname{dist}_{G}(u, v) \leq k$. Since $u \in U$ and $v \in V$, clearly $\operatorname{dist}_{G}(u, v)$ is odd. Thus we have the theorem by the two lemmas.

In what follows, we assume $u \in U$ and $v \in V$. We also assume that $G$ is connected. It is easy to see that if Theorem 3.2 is valid for connected graphs, then it is also valid for disconnected graphs.

Lemma 3.4 If $\operatorname{dist}_{G}(u, v) \leq k$, then $I_{u}(k) \cap I_{v}(k) \neq \emptyset$.

Proof: Without loss of generality, we assume $\ell_{u} \leq \ell_{v}$. Since $\operatorname{dist}_{G}(u, v)$ is odd and at most $k$, it holds that $\ell_{v} \leq r_{u}(k)$ from the definition of $r_{u}(k)$. Hence $\ell_{v} \in\left[\ell_{u}, r_{u}(k)\right]=I_{u}(k)$, and thus $I_{u}(k) \cap I_{v}(k) \neq \emptyset$.

Lemma 3.5 If $I_{u}(k) \cap I_{v}(k) \neq \emptyset$, then $\operatorname{dist}_{G}(u, v) \leq k$.

Proof: Without loss of generality, we assume $\ell_{u} \leq \ell_{v}$. By this assumption and $I_{u}(k) \cap I_{v}(k) \neq \emptyset$, it holds that $\ell_{u} \leq \ell_{v} \leq r_{u}(k)$. Let $v^{\prime} \in V$ be a vertex such that $\operatorname{dist}_{G}\left(u, v^{\prime}\right) \leq k$ and $r_{u}(k)=\ell_{v^{\prime}}$. Let $P=\left(u_{0}, v_{1}, u_{2}, \ldots, u_{2 d}, v_{2 d+1}\right)$ denote a shortest $u-v^{\prime}$ path in $G$, where $u_{0}=u$ and $v_{2 d+1}=v^{\prime}$. Clearly, $2 d+1 \leq k$. If $\left\{v, u_{i}\right\} \in E(G)$ for some $i$, there is a $u-v$ path $\left(u_{0}, v_{1}, u_{2} \ldots, v_{i-1}, u_{i}, v\right)$ of length $i+1 \leq 2 d+1 \leq k$ in $G$ and this implies that $\operatorname{dist}_{G}(u, v) \leq k$. Hence, we assume there is no such index $i$. Then, the following claim holds.

Claim 3.6 There is some index $j$ such that $I_{v} \subseteq I_{v_{j}}$.

Proof (Claim 3.6): Since $I_{v}$ does not intersect with any $I_{u_{i}}$, we can partition $U \cap V(P)$ into two parts $L(P)=\left\{u_{i} \mid r_{u_{i}}<\ell_{v}\right\}$ and $R(P)=\left\{u_{i} \mid r_{v}<\ell_{u_{i}}\right\}$. By the assumption $\ell_{u} \leq \ell_{v}$ and $\{u, v\}=\left\{u_{0}, v\right\} \notin E(G)$, it holds that $r_{u}<\ell_{v}$. Furthermore, since $\ell_{v} \leq r_{u}(k)=\ell_{v^{\prime}}$ and $\left\{u_{2 d}, v^{\prime}\right\}=$ $\left\{u_{2 d}, v_{2 d+1}\right\} \in E(G)$, we have $\ell_{v} \leq \ell_{v^{\prime}} \leq r_{u_{2 d}}$. Because $\left\{u_{2 d}, v\right\} \notin E(G)$, it holds that $r_{v}<\ell_{u_{2 d}}$. Hence, both $L(P)$ and $R(P)$ are non-empty since $u \in L(P)$ and $u_{2 d} \in R(P)$. Since $P$ is a connected subgraph of $G$, there is a vertex $v_{j}$ that has neighbors both in $L(P)$ and in $R(P)$. Then, the corresponding interval $I_{v_{j}}$ contains $I_{v}$ (see Fig. 11).

Since $G$ is connected, $v$ has a neighbor $u^{\prime}$ in $G$. Thus, we have $I_{v} \cap I_{u^{\prime}} \neq \emptyset$, which implies $I_{u^{\prime}} \cap I_{v_{j}} \neq \emptyset$. It is easy to see that $\left(u_{0}, v_{1}, \ldots, u_{j-1}, v_{j}, u^{\prime}, v\right)$ is a $u-v$ path of length $j+2$, where $j-1 \leq 2 d-2$. Therefore, the lemma holds. 


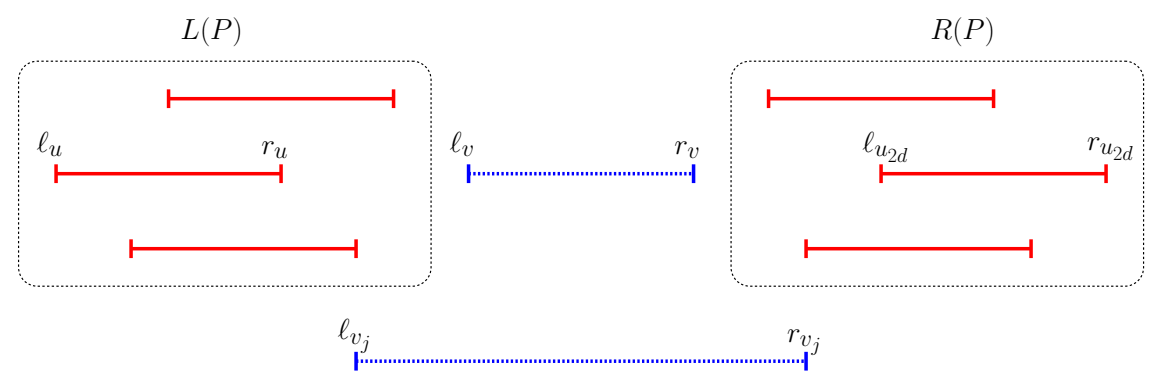

Fig. 1: The bridge interval $I_{v_{j}}$ between $L(P)$ and $R(P)$.

\subsection{Bipartite powers of bipartite permutation graphs}

Next we prove Theorem 3.3 An asteroidal triple is a set of three pairwise non-adjacent vertices such that there is a path between any pair of them avoiding the neighbors of the third. If a graph has no asteroidal triple, then it is AT-free. We use the following characterization of bipartite permutation graphs.

Theorem 3.7 (Hell and Huang (2004)) A bipartite graph is a permutation graph if and only if it is ATfree.

By the theorem above, it is sufficient to show the following lemma. Our proof is inspired by the proof of Raychaudhuri (1987), who proved that the $k$ th power of an AT-free graph is also AT-free.

Lemma 3.8 If $G$ is bipartite and AT-free, then so is $G^{[k]}$ for any odd $k \in \mathbb{Z}^{+}$.

Proof: Let $v_{1}, v_{2}, v_{3}$ be any pairwise non-adjacent triple of vertices in $G$. Without loss of generality, assume that any $v_{1}-v_{2}$ path in $G$ passes through a neighbor of $v_{3}$. We shall prove that any $v_{1}-v_{2}$ path in $G^{[k]}$ passes through a neighbor of $v_{3}$.

Let $P=\left(u_{1}, u_{2}, \ldots, u_{p}\right)$ be a $v_{1}-v_{2}$ path in $G^{[k]}$ such that $u_{1}=v_{1}$ and $u_{p}=v_{2}$. For $1 \leq i \leq$ $p-1$, let $P\left(u_{i}, u_{i+1}\right)$ denote a shortest $u_{i}-u_{i+1}$ path in $G$. Replacing every edge $\left\{u_{i}, u_{i+1}\right\}$ in $P$ by the corresponding path $P\left(u_{i}, u_{i+1}\right)$, we obtain a walk $W_{P}$ in $G$. Clearly, $W_{P}$ contains a $v_{1}-v_{2}$ path in $G$, and hence, $N_{G}\left(v_{3}\right) \cap V\left(W_{P}\right) \neq \emptyset$. Let $w \in N_{G}\left(v_{3}\right) \cap V\left(W_{P}\right)$. Assume, without loss of generality, $w \in V\left(P\left(u_{i}, u_{i+1}\right)\right)$ for some $i, 1 \leq i \leq p-1$. Since $\left\{u_{i}, u_{i+1}\right\} \in E\left(G^{[k]}\right), \operatorname{dist}_{G}\left(u_{i}, u_{i+1}\right)$ is odd and at most $k$. If $w \in\left\{u_{i}, u_{i+1}\right\}$, then $N_{G^{[k]}}\left(v_{3}\right) \cap V(P) \neq \emptyset$. Thus, we assume $w \notin\left\{u_{i}, u_{i+1}\right\}$. Since $P\left(u_{i}, u_{i+1}\right)$ is a shortest $u_{i}-u_{i+1}$ path in $G$ and $w \in V\left(P\left(u_{i}, u_{i+1}\right)\right)$, we have $\operatorname{dist}_{G}\left(u_{i}, w\right)+$ $\operatorname{dist}_{G}\left(w, u_{i+1}\right)=\operatorname{dist}_{G}\left(u_{i}, u_{i+1}\right)$. Thus, $\operatorname{dist}_{G}\left(u_{i}, u_{i+1}\right) \leq k$ and $w \notin\left\{u_{i}, u_{i+1}\right\}$ together imply $\operatorname{dist}_{G}\left(u_{i}, w\right)<k$ and $\operatorname{dist}_{G}\left(w, u_{i+1}\right)<k$. Hence, $\operatorname{dist}_{G}\left(v_{3}, u_{i}\right) \leq k$ and $\operatorname{dist}_{G}\left(v_{3}, u_{i+1}\right) \leq k$. Since $\operatorname{dist}_{G}\left(u_{i}, u_{i+1}\right)$ is odd, one of $\operatorname{dist}_{G}\left(u_{i}, w\right)$ and $\operatorname{dist}_{G}\left(w, u_{i+1}\right)$ is also odd.

Without loss of generality, we assume $\operatorname{dist}_{G}\left(u_{i}, w\right)$ is odd, and $\operatorname{dist}_{G}\left(w, u_{i+1}\right)$ is even. Then, $G$ has a $v_{3}-u_{i+1}$ path with length $\operatorname{dist}_{G}\left(w, u_{i+1}\right)+1$, which is odd and at most $k$. Since $G$ is bipartite, $G$ cannot have any $v_{3}-u_{i+1}$ path of even length. Hence $\operatorname{dist}_{G}\left(v_{3}, u_{i+1}\right)$ is odd and at most $k$. This implies that $u_{i+1} \in N_{G^{[k]}}\left(v_{3}\right)$ and therefore, $N_{G^{[k]}}\left(v_{3}\right) \cap P \neq \emptyset$. 


\section{Recognizing bipartite powers of bigraphs is hard}

Powers of graphs are studied also in computational complexity aspects. Motwani and Sudan (1994) first showed that given a graph $G$, it is NP-complete to decide whether there exists a graph $H$ such that $H^{2}=G$. Following their result, several related results have been obtained (Chang et al. (2006); Farzad et al. (2009); Kearney and Corneil (1998); Lau (2006); Lau and Corneil (2004); Le and Nguyen (2010); Lin and Skiena (1995)). Recently, Le and Nguyen (2010) have shown the following hardness result.

Theorem 4.1 (Le and Nguyen (2010)) For any fixed $k \geq 2$ and given a graph $G$, it is NP-complete to determine whether there exists a graph $H$ such that $H^{k}=G$.

In this section, we prove that the problem of recognizing bipartite powers is also hard.

Theorem 4.2 For any fixed odd $k \geq 3$ and given a bipartite graph $G$, it is NP-complete to determine whether there exists a bipartite graph $H$ such that $H^{[k]}=G$.

The problem is clearly in NP. Thus, it is sufficient to show that the problem is NP-hard. We reduce SET SPLiTTING, a well-known NP-complete problem, to ours.

Problem: Set Splitting (Garey and Johnson, 1979, SP4)

Instance: Collection $C$ of non-empty subsets of a finite set $S$.

Question: Is there a partition of $S$ into two subsets $S_{1}$ and $S_{2}$ such that no subset in $C$ is entirely contained in either $S_{1}$ or $S_{2}$ ?

Without loss of generality, we assume that every element of $S$ belongs to at least one set in $C$.

\subsection{An auxiliary lemma}

Before presenting the reduction, we show a useful lemma. In their proof of NP-hardness, Le and Nguyen (2010) used the following tail structure presented first by Motwani and Sudan (1994) and generalized later by $\operatorname{Lau}(2006)$. Note that for two sets $A$ and $B$, we mean by $A \subset B$ that $A \subseteq B$ and $A \neq B$; that is, $A$ is properly included in $B$.

Lemma $4.3\left(\right.$ Lau (2006)) Let $G$ be a connected graph with $\left\{v_{1}, \ldots, v_{k+1}\right\} \subset V(G)$ where $N_{G}\left(v_{1}\right)=$ $\left\{v_{2}, \ldots, v_{k+1}\right\}$, and $N_{G}\left(v_{i}\right) \subset N_{G}\left[v_{i+1}\right]$ for all $1 \leq i \leq k$. If $H^{k}=G$ for some graph $H$, then

1. $N_{H}\left(v_{1}\right)=\left\{v_{2}\right\}, N_{H}\left(v_{i}\right)=\left\{v_{i-1}, v_{i+1}\right\}$ for all $2 \leq i \leq k$, and

2. $N_{H}\left(v_{k+1}\right) \backslash\left\{v_{k}\right\}=N_{G}\left(v_{2}\right) \backslash\left\{v_{1}, v_{2}, \ldots, v_{k+1}\right\}$.

The tail structure played an important role in the proofs of NP-hardness of recognizing powers of graphs (Lau (2006); Le and Nguyen (2010); Motwani and Sudan (1994)). Although we cannot use the structure directly, we show the following similar structure for bipartite powers.

Lemma 4.4 Let $k \geq 3$ be odd, and $G$ be a connected bipartite graph with $\left\{v_{0}, \ldots, v_{k+1}\right\} \subset V(G)$ where $N_{G}\left(v_{0}\right)=\left\{v_{1}, v_{3}, \ldots, v_{k-2}, v_{k}\right\}, N_{G}\left(v_{1}\right)=\left\{v_{0}, v_{2}, \ldots, v_{k-1}, v_{k+1}\right\}$, and $N_{G}\left(v_{i}\right) \subset N_{G}\left(v_{i+2}\right)$ for $0 \leq i \leq k-1$. If $H^{[k]}=G$ for some bipartite graph $H$, then

1. $N_{H}\left(v_{0}\right)=\left\{v_{1}\right\}, N_{H}\left(v_{i}\right)=\left\{v_{i-1}, v_{i+1}\right\}$ for $1 \leq i \leq k$, and

2. $N_{H}\left(v_{k+1}\right) \backslash\left\{v_{k}\right\}=N_{G}\left(v_{2}\right) \backslash\left\{v_{0}, v_{1}, \ldots, v_{k+1}\right\}$. 
To prove Lemma 4.4 we need some definitions. A vertex $u \in U$ in a bipartite graph $G=(U, V ; E)$ is bipartite universal if $N_{G}(u)=V$. We denote by $N_{H}^{d}(v)$ the set of vertices in $H$ such that the distance from $v$ is exactly $d$; that is, $N_{H}^{d}(v)=\left\{u \in V(H) \mid \operatorname{dist}_{H}(u, v)=d\right\}$.

Proof (Lemma 4.4): First, observe that the vertices $v_{0}$ and $v_{1}$ are not bipartite universal in $G$.

Since $v_{0}$ is not bipartite universal in $G=H^{[k]}$ and $G$ is connected, there is a vertex $u \in V(H)$ such that $\operatorname{dist}_{H}\left(v_{0}, u\right)$ is finite and greater than $k$. This implies that $N_{H}^{2 i+1}\left(v_{0}\right) \neq \emptyset$ for all $0 \leq i \leq(k-1) / 2$. Moreover, since $\left|N_{G}\left(v_{0}\right)\right|=\left|\left\{v_{1}, v_{3}, \ldots, v_{k-2}, v_{k}\right\}\right|=(k+1) / 2$, it follows $\left|N_{H}^{2 i+1}\left(v_{0}\right)\right|=1$ for all $0 \leq i \leq(k-1) / 2$. Then, the assumption $N_{G}\left(v_{i}\right) \subset N_{G}\left(v_{i+2}\right)$ implies that $N_{H}^{2 i+1}\left(v_{0}\right)=\left\{v_{2 i+1}\right\}$ for all $0 \leq i \leq(k-1) / 2$ (see Fig. 2).

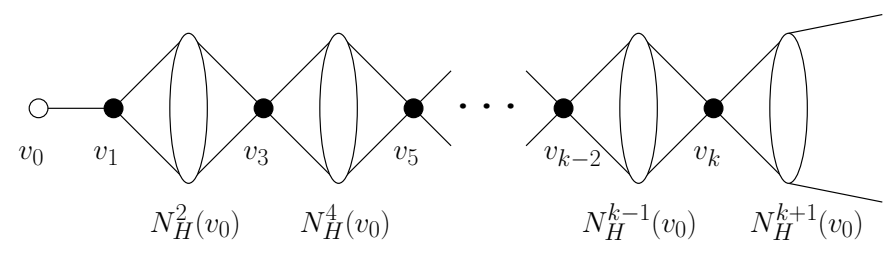

Fig. 2: Partial tail structure of vertices.

Next we apply a similar argument to $v_{1}$. Since $v_{1}$ is not bipartite universal in $G, N_{H}^{2 i+1}\left(v_{1}\right) \neq \emptyset$ for all $0 \leq i \leq(k-1) / 2$. Since $N_{H}\left(v_{0}\right)=\left\{v_{1}\right\}$ and $\left|N_{G}\left(v_{1}\right)\right|=\left|\left\{v_{0}, v_{2}, \ldots, v_{k-1}, v_{k+1}\right\}\right|=(k+1) / 2+1$, it follows $\left|N_{H}\left(v_{1}\right)\right|=2$ and $\left|N_{H}^{2 i+1}\left(v_{1}\right)\right|=1$ for all $1 \leq i \leq(k-1) / 2$. By the assumption $N_{G}\left(v_{i}\right) \subset$ $N_{G}\left(v_{i+2}\right)$, we have $N_{H}\left(v_{1}\right)=\left\{v_{0}, v_{2}\right\}$ and $N_{H}^{2 i+1}\left(v_{1}\right)=\left\{v_{2 i+2}\right\}$ for $1 \leq i \leq(k-1) / 2$ (see Fig. 3 . Thus the first property holds. The second property immediately follows from the first one.

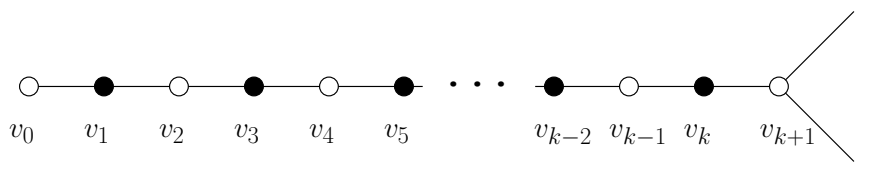

Fig. 3: Tail structure of vertices.

\subsection{Reduction}

Let $(S, C)$ be an instance of Set Splitting, where $S=\left\{u_{1}, \ldots, u_{n}\right\}$ is the ground set, and $C=$ $\left\{c_{1}, \ldots, c_{m}\right\}$ is a set of non-empty subsets of $S$. For any fixed odd $k \geq 3$, we construct a graph $G$ from $(S, C)$. The vertex set $V(G)$ of $G$ consists of the following vertices:

- Element vertices $u_{i}$ for all element $u_{i} \in S$;

- Subset vertices $c_{j}^{0}, \ldots, c_{j}^{k+1}$ for all $c_{j} \in C$; and

- Partition vertices $S_{1}^{0}, \ldots, S_{1}^{k-2}$ and $S_{2}^{0}, \ldots, S_{2}^{k-2}$. 
The edge set $E(G)$ of $G$ consists of the following edges: For each subset $c_{j} \in C$,

- $c_{j}^{p}$ is adjacent to $c_{j}^{q}$ if $p \not \equiv q(\bmod 2)$,

- $c_{j}^{p}$ is adjacent to $c_{j^{\prime}}^{q}$ if $p+q \leq k-4$ and $p \not \equiv q(\bmod 2)$;

- $c_{j}^{p}$ is adjacent to $c_{j^{\prime}}^{q}$ if $c_{j} \cap c_{j^{\prime}} \neq \emptyset$ and $p+q=k-2$,

- $c_{j}^{p}$ is adjacent to all $u_{i} \in S$ if $p$ is even and $0 \leq p \leq k-3$,

- $c_{j}^{k-1}$ is adjacent to each $u_{i} \in c_{j}$,

- $c_{j}^{p}$ is adjacent to $S_{1}^{q}$ and $S_{2}^{q}$ if $p+q \leq k-2$ and $p \not \equiv q(\bmod 2)$,

For each element $u_{i} \in S$,

- $u_{i}$ is adjacent to $S_{1}^{0}, S_{1}^{2}, \ldots, S_{1}^{k-3}$ and $S_{2}^{0}, S_{2}^{2}, \ldots, S_{2}^{k-3}$;

For each $i \in\{1,2\}$,

- $S_{i}^{p}$ is adjacent to $S_{i}^{q}$ if $p \not \equiv q(\bmod 2)$,

- $S_{1}^{p}$ and $S_{2}^{q}$ are adjacent if $p+q \leq k-4$ and $p \not \equiv q(\bmod 2)$.

\subsection{Equivalence}

Now we prove that $(S, C)$ has a desired partition if and only if there exists a bipartite graph $H$ such that $H^{[k]}=G$.

Lemma 4.5 If there exists a partition of $S$ into two disjoint subsets $S_{1}$ and $S_{2}$ such that each subset in $C$ intersects both $S_{1}$ and $S_{2}$, then there exists a bipartite graph $H$ such that $G=H^{[k]}$.

Proof: Let $V(H)=V(G)$. We define $E(H)$ as follows (see Fig. 44).

- For each $c_{j} \in C,\left\{c_{j}^{i}, c_{j}^{i+1}\right\} \in E(H)$ for $0 \leq i \leq k$, and $\left\{c_{j}^{0}, u_{i}\right\} \in E(H)$ for each $u_{i} \in c_{j}$.

- For each $u_{i} \in S,\left\{u_{i}, S_{j}^{0}\right\} \in E(H)$ if $u_{i} \in S_{j}$.

- For $j \in\{1,2\},\left\{S_{j}^{i}, S_{j}^{i+1}\right\} \in E(H)$ for $0 \leq i \leq k-3$.

It is a routine exercise to verify that $G=H^{[k]}$.

Lemma 4.6 If there is a bigraph $H$ such that $H^{[k]}=G$, then there exists a partition of $S$ into two disjoint subsets $S_{1}$ and $S_{2}$ such that each subset in $C$ intersects both $S_{1}$ and $S_{2}$.

Proof: We construct a partition $\left(S_{1}, S_{2}\right)$ of $S$ such that $S_{1}=\left\{u_{i} \mid\left\{u_{i}, S_{1}^{0}\right\} \in E(H)\right\}$ and $S_{2}=S \backslash S_{1}$. We show that $\left(S_{1}, S_{2}\right)$ is a good partition; that is, $c_{j} \cap S_{i} \neq \emptyset$ for each $c_{j} \in C$ and $i \in\{1,2\}$. To this end, we show that

1. for any $c_{j} \in C$ and $\ell \in\{1,2\}$, some $u_{i} \in c_{j}$ has $S_{\ell}^{0}$ as a neighbor in $H$, and 


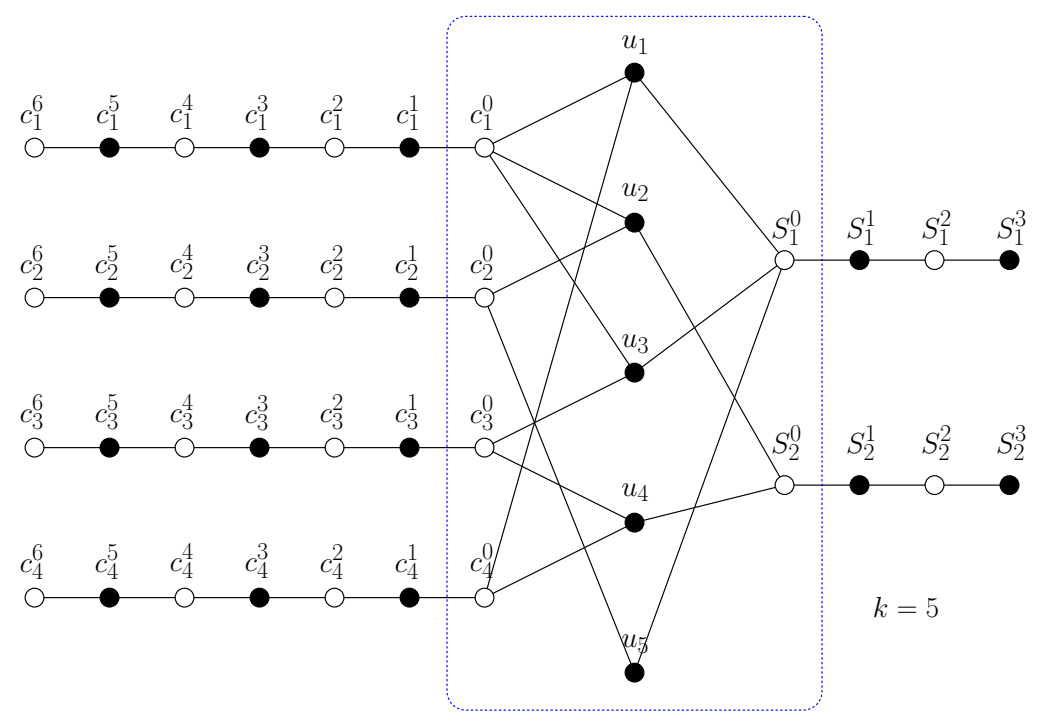

Fig. 4: An example $(k=5)$.

2. no $u_{i} \in S$ has both $S_{1}^{0}$ and $S_{2}^{0}$ as neighbors in $H$.

They will conclude the proof. Let $c_{j} \in C$. From Lemma $4.4 . N_{H}\left(c_{j}^{k+1}\right)=\left\{c_{j}^{k}\right\}, N_{H}\left(c_{j}^{i}\right)=\left\{c_{j}^{i+1}, c_{j}^{i-1}\right\}$ for $1 \leq i \leq k$, and $N_{H}\left(c_{j}^{0}\right)=\left\{c_{j}^{1}\right\} \cup\left\{u_{i} \mid u_{i} \in c_{j}\right\}$. Obviously, $\operatorname{dist}_{H}\left(c_{j}^{p}, c_{j}^{q}\right)=|p-q|$. For $v \notin\left\{c_{j}^{0}, \ldots, c_{j}^{k+1}\right\}$, every $c_{j}^{p}-v$ path in $H$ has $c_{j}^{q}$ as an internal node if $p>q$. Hence, $\operatorname{dist}_{H}\left(c_{j}^{p}, v\right)=$ $p-q+\operatorname{dist}_{H}\left(c_{j}^{q}, v\right)$. Note that this also holds when $p \leq q$.

Claim 4.7 For $\ell \in\{1,2\}$ and $a, b \geq 0$, $\operatorname{dist}_{H}\left(c_{j}^{a}, S_{\ell}^{b}\right)=a+b+2$.

Proof (Claim 4.7): First, we show that $\operatorname{dist}_{H}\left(c_{j}^{k-b-2}, S_{\ell}^{b}\right)=k$.

Since $\left\{c_{j}^{k-b-2}, S_{\ell}^{b}\right\} \in E(G)$, $\operatorname{dist}_{H}\left(c_{j}^{k-b-2}, S_{\ell}^{b}\right)$ is odd and at most $k$. Clearly, $\operatorname{dist}_{H}\left(c_{j}^{k-b}, S_{\ell}^{b}\right)$ is also odd. Thus $\left\{c_{j}^{k-b}, S_{\ell}^{b}\right\} \notin E(G)$ implies that

$$
\operatorname{dist}_{H}\left(c_{j}^{k-b}, S_{\ell}^{b}\right)=2+\operatorname{dist}_{H}\left(c_{j}^{k-b-2}, S_{\ell}^{b}\right)>k .
$$

Hence, $\operatorname{dist}_{H}\left(c_{j}^{k-b-2}, S_{\ell}^{b}\right)=k$ since $k$ is odd. Recall that $\operatorname{dist}_{H}\left(c_{j}^{p}, v\right)=p-q+\operatorname{dist}_{H}\left(c_{j}^{q}, v\right)$. Therefore,

$$
\operatorname{dist}_{H}\left(c_{j}^{a}, S_{\ell}^{b}\right)=a-(k-b-2)+\operatorname{dist}_{H}\left(c_{j}^{k-b-2}, S_{\ell}^{b}\right)=a+b+2 .
$$

Thus, the claim holds.

Let $\ell \in\{1,2\}$. From Claim 4.7 , we have $\operatorname{dist}_{H}\left(c_{j}^{0}, S_{\ell}^{0}\right)=2$. Since $N_{H}\left(c_{j}^{0}\right)=\left\{c_{j}^{1}\right\} \cup\left\{u_{i} \mid u_{i} \in c_{j}\right\}$ and $N_{H}\left(c_{j}^{1}\right)=\left\{c_{j}^{2}, c_{j}^{0}\right\}$, some $u_{i} \in c_{j}$ must be adjacent to $S_{\ell}^{0}$ in $H$. Thus, we have the first property. To show the second property, we use the following fact. 
Claim 4.8 $\operatorname{dist}_{H}\left(S_{2}^{0}, S_{2}^{k-2}\right)=k-2$.

Proof (Claim 4.8): By Claim 4.7, any shortest $c_{j}^{0}-S_{2}^{k-2}$ path in $H$ is of length $k$. Let $P=\left(p_{0}, p_{1}, \ldots, p_{k}\right)$ be such a path, where $p_{0}=c_{j}^{0}$ and $p_{k}=S_{2}^{k-2}$. It is easy to see that $p_{1}=u_{i}$ for some $u_{i} \in c_{j}$. Also, it is not difficult to see that either $p_{2}=c_{j^{\prime}}^{0}$ for some $c_{j^{\prime}} \in C$, or $p_{2}=S_{\ell}^{x}$ for some $x \in\{0, \ldots, k-2\}$ and $\ell \in\{1,2\}$. If $p_{2}=c_{j^{\prime}}^{0}$, then $\operatorname{dist}_{H}\left(c_{j^{\prime}}^{0}, S_{2}^{k-2}\right)=k-2$. This contradicts Claim 4.7 Hence, we have $p_{2}=S_{\ell}^{x}$. Since $\left(c_{j}^{0}, u_{i}, S_{\ell}^{x}\right)$ is a shortest path in $H$, $\operatorname{dist}_{H}\left(c_{j}^{0}, S_{\ell}^{x}\right)=2$. Thus we have $x=0$ from Claim 4.7 If $p_{2}=S_{1}^{0}$, then $\left(p_{2}, \ldots, p_{k}\right)$ is a $S_{1}^{0}-S_{2}^{k-2}$ path of length $k-2$. So, $\operatorname{dist}_{H}\left(S_{1}^{0}, S_{2}^{k-2}\right)=k-2$. This contradicts $\left\{S_{1}^{0}, S_{2}^{k-2}\right\} \notin E(G)=E\left(H^{[k]}\right)$. Hence, we can conclude that $p_{2}=S_{2}^{0}$, and thus $\left(p_{2}, \ldots, p_{k}\right)$ is a shortest $S_{2}^{0}-S_{2}^{k-2}$ path of length $k-2$ in $H$.

Now we prove the second property. Suppose some $u_{i} \in c_{j}$ has both $S_{1}^{0}$ and $S_{2}^{0}$ as neighbors in $H$. Then, $\operatorname{dist}_{H}\left(S_{1}^{0}, S_{2}^{0}\right)=2$. Since $\operatorname{dist}_{H}\left(S_{2}^{0}, S_{2}^{k-2}\right)=k-2$ by Claim 4.8 .

$$
\operatorname{dist}_{H}\left(S_{1}^{0}, S_{2}^{k-2}\right) \leq \operatorname{dist}_{H}\left(S_{1}^{0}, S_{2}^{0}\right)+\operatorname{dist}_{H}\left(S_{2}^{0}, S_{2}^{k-2}\right)=k .
$$

Since there is a $S_{1}^{0}-S_{2}^{k-2}$ path of odd length in $G$ and because $G$ is bipartite, $\operatorname{dist}_{H}\left(S_{1}^{0}, S_{2}^{k-2}\right)$ is odd. Hence, $\left\{S_{1}^{0}, S_{2}^{k-2}\right\} \in E\left(H^{[k]}\right)$. This contradicts $\left\{S_{1}^{0}, S_{2}^{k-2}\right\} \notin E(G)=E\left(H^{[k]}\right)$.

\section{References}

G. Agnarsson, R. Greenlaw, and M. M. Halldórsson. On powers of chordal graphs and their coloring. Congr. Numer., 144:4165, 2000.

L. S. Chandran and R. Mathew. Bipartite powers of $k$-chordal graphs, 2012. Preprint available at http:// arxiv.org/abs/1108.0277.

L. S. Chandran, M. Francis, and R. Mathew. Chordal bipartite graphs with high boxicity. Graphs Combin., 27:353-362, 2011.

M.-S. Chang, M.-T. Ko, and H.-I. Lu. Linear-time algorithms for tree root problems. In SWAT 2006, volume 4059 of Lecture Notes in Comput. Sci., pages 411-422, 2006.

M. Chen and G. J. Chang. Families of graphs closed under taking powers. Graphs Combin., 17:207-212, 2001.

E. Dahlhaus and P. Duchet. On strongly chordal graphs. Ars Combin., 24B:23-30, 1987.

B. Farzad, L. C. Lau, V. B. Le, and N. N. Tuy. Computing graph roots without short cycles. In STACS 2009, volume 3 of LIPIcs, pages 397-408, 2009.

M. R. Garey and D. S. Johnson. Computers and Intractability: A Guide to the Theory of NP-Completeness. Freeman, 1979.

P. Hell and J. Huang. Interval bigraphs and circular arc graphs. J. Graph Theory, 46:313-327, 2004.

P. E. Kearney and D. G. Corneil. Tree powers. J. Algorithms, 29:111-131, 1998. 
L. C. Lau. Bipartite roots of graphs. ACM Trans. Algorithms, 2:178-208, 2006.

L. C. Lau and D. G. Corneil. Recognizing powers of proper interval, split, and chordal graphs. SIAM J. Discrete Math., 18:83-102, 2004.

V. B. Le and N. T. Nguyen. Hardness results and efficient algorithms for graph powers. In WG 2009, volume 5911 of Lecture Notes in Comput. Sci., pages 238-249, 2010.

Y.-L. Lin and S. S. Skiena. Algorithms for square roots of graphs. SIAM J. Discrete Math., 8:99-118, 1995.

A. Lubiw. Doubly lexical ordering of matrices. SIAM J. Comput., 16(5):854-879, 1987.

R. Motwani and M. Sudan. Computing roots of graphs is hard. Discrete Appl. Math., 54:81-88, 1994.

H. Müller. Recognizing interval digraphs and interval bigraphs in polynomial time. Discrete Appl. Math., 78(1-3):189-205, 1997. Erratum is available at http://www.comp.leeds.ac.uk/hm/pub/node1.html.

E. Prisner. Graph Dynamics. Longman, 1995.

A. Raychaudhuri. On powers of interval and unit interval graphs. Congr. Numer., 59:235-242, 1987.

A. Raychaudhuri. On powers of strongly chordal and circular arc graphs. Ars Combin., 34:147-160, 1992. 